\title{
Nuclear peroxiredoxine: a promising target for drug \& diagnostic solutions of malaria
}

\begin{abstract}
During development inside the erythrocytes, malaria parasites plasmodium have to face constant attack of oxidative stress build up by reactive oxygen and nitrogen species produced exogenously \& endogenously. Plasmodium utilizes a unique antioxidant defense system for their survival against this oxidative attack. A recent report has characterized an unusual nuclear peroxiredoxine in Plasmodium falciparum, PfnPrx that has a broad specificity of substrate and having the ability to reduce peroxides from simple to complex one. Study revealed its association with chromatin of the parasite in genome wide manner which suggests an essential role in protection of nuclear DNA and expression throughout the erythrocytic stage. This mini review will discuss about the possibilities of $P$. falciparum nuclear peroxiredoxine as a potential target for drug discovery and development of diagnostic products of Malaria.
\end{abstract}

Keywords: plasmodium falciparum nuclear peroxiredoxine, oxidative stress, drug, diagnosis, malaria
Volume 3 Issue 2 - 2016

\author{
Navin Kumar,' Sona Mishra ${ }^{2}$ \\ 'Epidemiology \& Clinical Research Division, National Institute of \\ Malaria Research, India \\ ${ }^{2}$ Department of Animal Biotechnology, Gautam Buddha \\ University, India
}

\begin{abstract}
Correspondence: Navin Kumar, Epidemiology \& Clinica Research Division, National Institute of Malaria Research (ICMR), Sector - 8 Dwarka, New Delhi - I I0077, India, Tel +917274802736, Email navinjh@gmail.com
\end{abstract}

Received: August 30, 2016 | Published: December 02, 2016
Abbreviations: PfnPrx, plasmodium falciparum nuclear peroxiredoxine; Prx, peroxiredoxin; RDTs, rapid diagnostic tests; ACTs, artemisinin based combination therapy

\section{Introduction}

Malaria has been always a major parasitic disease worldwide with millions of confirmed cases and deaths per year. ${ }^{1}$ Gradually increase in drug resistance against an effective Artemisinin based combination therapies ACTs which includes Sulphadoxine, Pyrimethamine \& conventional chloroquine advocated by recent reports ${ }^{2}$ and lack of vaccine have now demonstrated the need to identify new potential drug targets. During development inside the infected erythrocytes, parasites have to face constant oxidative attack from reactive oxygen and nitrogen species produced exogenously by the immune system of host in response to infection \& endogenously by the by-products resulting from the high multiplication of parasites hemoglobin degradation. ${ }^{3}$ To survive in oxidative stress environment, parasites adapt multiple biochemical pathway foe antioxidant defence and redox regulation., 4 Since Plasmodium don't have catalase and glutathione peroxidase, they heavily relies on Peroxiredoxin to cope up oxidative attack. $^{6}$

\section{Nuclear peroxiredoxin, as a target for drug discovery}

Peroxiredoxins (Prx) belong to anti-oxidant family which protects the cell from metabolically produced reactive oxygen and nitrogen species. So, it's an important part of antioxidant machinery of cell. Usually it contains at least one cysteine residue in their active site, called as peroxidatic cysteine due to its ability to react with peroxide substrate. It's a defining feature of this group of enzymes referred as AhpC-TSA family4. Beside the other cellular processes like apoptosis, cell proliferation \& differentiation, it also known as redox sensors in response to oxidative damage 11 Generally, organism have multiple peroxiredoxine found in different location in cell such as nucleus, cytosol, mitochondria and peroxisomes. ${ }^{7}$ Same is also applicable for plasmodium. Till date, five malarial peroxiredoxine have been studied i.e. PfTrx-Px2 in mitochondria, PfTrx-Px1 \& PfTrx-Px3 in cytosol, PfAOP in apicoplast and finally a glutathione dependent like peroxidase ${ }^{8-10} \mathrm{~A}$ recent study has reported that the additional protein with a conserved AhpC-TSA domain, earlier called as MCP1 (merozoite capping protein-1) is exclusively localized in nucleus i.e. P. falciparum nuclear Peroxiredoxin (PfnPrx) ${ }^{11}$ Its association with the chromatin of parasite have been also demonstrated which suggests the potentiality of PfnPrx in the protection of nuclear genome, DNA repair etc. against oxidative damage. It has been also suggested that the PfnPrx may be essential in erythrocytic stage, as they have failed to generate knock out lines in P. falciparum or P. berghei despite of multiple attempts and success in generating tagged nPrx-GFP fusion cell lines. ${ }^{11}$ So, it could potentially be used as a target for the development of inhibitor based therapy.

\section{Nuclear peroxiredoxin, as a target for rapid diagnosis of malaria}

Early, accurate diagnosis and prompt treatment are one of the important strategies for malaria control. Detection of malaria parasites by microscopic examination of blood smears remains the gold standard but may not be easily available especially in remote areas. The development of alternative diagnostic method like rapid diagnostic test, (RDTs) has made it possible to provide rapid and accurate detection of malaria parasites in remote areas where microscopy facility is not available. Several factors affect the performance of malaria RDTs which include test factors \& parasite factors but genetic variability ${ }^{12,13}$ and genetic deletion of these diagnostic antigens have been also questioned their sensitivity \& reliability. ${ }^{14}$ So, there is also an urgent need to develop new reliable diagnostic target of malaria parasite. For this point of view, P. falciparum nuclear Peroxiredoxin (PfnPrx) may be used as an ideal candidate to explore the diagnostic future as it has been also suggested in recent study that $P$. falciparum nuclear Peroxiredoxin (PfnPrx) express throughout the blood stage of parasite. ${ }^{14}$ So, development of monoclonal antibody that target PfnPrx for the diagnostic solutions of malaria would be also useful as it's essentiality during erythrocytic stage of parasite has been also advocated. 


\section{Acknowledgements}

None.

\section{Conflict of interest}

The author declares no conflict of interest.

\section{References}

1. Snow RW, Guerra CA, Noor AM, et al. The global distribution of clinical episodes of Plasmodium falciparum malaria. Nature. 2005;434(7030):214-217.

2. White NJ. Qinghaosu (artemisinin):the price of success. Science. 2008;320(5874):330-334.

3. Wood ZA, Schröder E, Robin Harris J, et al. Structure, mechanism and regulation of peroxiredoxins. Trends Biochem Sci. 2003;28(1):32-40.

4. Rouhier N, Gelhaye E, Jacquot JP. Glutaredoxin-dependent peroxiredoxin from poplar: protein-protein interaction and catalytic mechanism. $J$ Biol Chem. 2002;277(16):13609-13614.

5. Wood ZA, Poole LB, Hantgan RR, et al. Dimers to doughnuts:redoxsensitive oligomerization of 2-cysteine peroxiredoxins. Biochemistry. 2002;41(17):5493-5504.

6. Sztajer $\mathrm{H}$, Gamain B, Aumann $\mathrm{KD}$, et al. the putative glutathione peroxidase gene of Plasmodium falciparum codes for a thioredoxin peroxidase. J Biol Chem. 2001;276(10):7397-7403.

7. Zykova TA, Zhu F, Vakorina TI, et al. T-LAK cell-originated protein kinase (TOPK) phosphorylation of Prx1 at Ser-32 prevents UVBinduced apoptosis in RPMI7951 melanoma cells through the regulation of Prx1 peroxidase activity. J Biol Chem. 2010;285(38):29138-29146.
8. Boucher IW, McMillan PJ, Gabrielsen M, et al. Structural and biochemical characterization of a mitochondrial peroxiredoxin from Plasmodium falciparum. Mol Microbiol. 2006;61(4):948-959.

9. Kawazu S, Tsuji N, Hatabu T, et al. Molecular cloning and characterization of a peroxiredoxin from the human malaria parasite Plasmodium falciparum. Mol Biochem Parasitol. 2000;109(2):165-169.

10. Krnajski Z, Walter RD, Müller S. Isolation and functional analysis of two thioredoxin peroxidases (peroxiredoxins) from Plasmodium falciparum. Mol Biochem Parasitol. 2001;113(2):303-308.

11. Richard D, Bartfai R, Volz J, et al. A genome-wide chromatin associated nuclear peroxiredoxine from the malaria parasites Plasmodium falciparum. J Biol Chem. 2011;286(13):11746-11755.

12. Baker J, Ho MF, Pelecanos A, et al. Global sequence variation in the histidine-rich proteins 2 and 3 of Plasmodium falciparum: implications for the performance of malaria rapid diagnostic tests. Malar $J$. 2010;9:129.

13. Kumar N, Singh JP, Pande V, et al. Genetic variation in histidine rich proteins among Indian Plasmodium falciparum population: possible cause of variable sensitivity of malaria rapid diagnostic tests. Malar $J$. 2012;11:298.

14. Kumar N, Pande V, Bhatt RM, et al. Genetic deletion of HRP2 and HRP3 in Indian Plasmodium falciparum population and false negative malaria rapid diagnostic test. Acta Trop. 2013;125(1):119-121. 